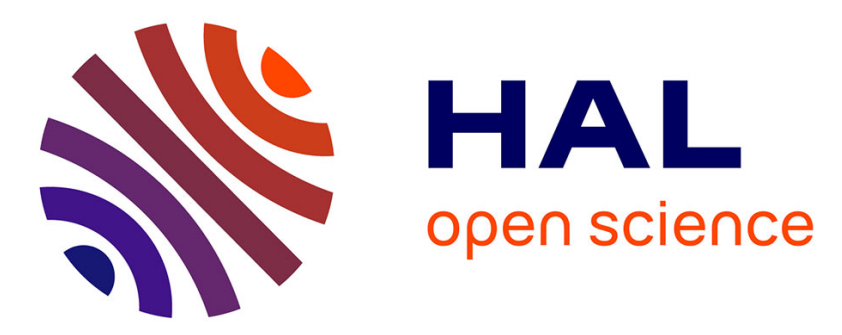

\title{
Nonlinear system identification of the dynamics of a vibro-impact beam: numerical results
}

Mehmet Kurt, Heng Chen, Young S. Lee, Michael Mcfarland, Lawrence A Bergman, Alexander F Vakakis

\section{- To cite this version:}

Mehmet Kurt, Heng Chen, Young S. Lee, Michael Mcfarland, Lawrence A Bergman, et al.. Nonlinear system identification of the dynamics of a vibro-impact beam: numerical results. Archive of Applied Mechanics, 2012, 82 (10-11), pp.1461-1479. 10.1007/s00419-012-0678-5 . hal-01620587

\section{HAL Id: hal-01620587 https://hal.science/hal-01620587}

Submitted on 22 Jan 2018

HAL is a multi-disciplinary open access archive for the deposit and dissemination of scientific research documents, whether they are published or not. The documents may come from teaching and research institutions in France or abroad, or from public or private research centers.
L'archive ouverte pluridisciplinaire HAL, est destinée au dépôt et à la diffusion de documents scientifiques de niveau recherche, publiés ou non, émanant des établissements d'enseignement et de recherche français ou étrangers, des laboratoires publics ou privés. 
Mehmet Kurt • Heng Chen • Young S. Lee •

D. Michael McFarland • Lawrence A. Bergman •

Alexander F. Vakakis

\title{
Nonlinear system identification of the dynamics of a vibro-impact beam: numerical results
}

\begin{abstract}
We study the dynamics of a cantilever beam with two rigid stops of certain clearances by performing nonlinear system identification (NSI) based on the correspondence between analytical and empirical slow-flow dynamics. The NSI method in this work can proceed in two directions: One for the numerical data obtained from a reduced-order model by means of the assumed-mode method, and the other for the experimental data measured at the same positions as in numerical simulations. This paper focuses on the analysis of the numerical data, providing qualitative comparison with some experimental results; the latter task will be discussed in detail in a companion paper. First, we perform empirical mode decomposition (EMD) on the acceleration responses measured at ten, almost evenly-spaced, spanwise positions along the beam leading to sets of intrinsic modal oscillators governing the vibro-impact dynamics at different time scales. In particular, the EMD analysis can separate any nonsmooth effects caused by vibro-impacts of the beam and the rigid stops from the smooth (elastodynamic) response, so that nonlinear modal interactions caused by vibro-impacts can be explored only with the remaining smooth components. Then, we establish nonlinear interaction models (NIMs) for the respective intrinsic modal oscillators, where the NIMs invoke slowly-varying forcing amplitudes that can be computed from empirical slow-flows. By comparing the spatio-temporal variations of the nonlinear modal interactions
\end{abstract}

\section{Kurt}

Department of Mechanical Science and Engineering, University of Illinois at Urbana-Champaign, 1206 W. Green Street, Urbana, IL 61801, USA

E-mail: kurt2@illinois.edu

H. Chen · Y. S. Lee (凶)

Department of Mechanical and Aerospace Engineering, New Mexico State University, 1040 S. Horseshoe Street, Las Cruces, NM 88003, USA

E-mail: younglee@nmsu.edu

H. Chen

E-mail: hengchen@nmsu.edu

D. M. McFarland · L. A. Bergman

Department of Aerospace Engineering, University of Illinois at Urbana-Champaign, 104 S. Wright Street, Urbana, IL 61801, USA

E-mail: dmmcf@illinois.edu

L. A. Bergman

E-mail: lbergman@illinois.edu

A. F. Vakakis

Department of Mechanical Science and Engineering, University of Illinois at Urbana-Champaign, 1206 W. Green Street, Urbana, IL 61801, USA

E-mail: avakakis@illinois.edu 
for the vibro-impact beam and those of the underlying linear model (i.e., the beam with no rigid constraints), we demonstrate that vibro-impacts significantly influence the lower-frequency modes, introducing spatial modal distortions, whereas the higher frequency modes tend to retain their linear dynamics between impacts. We introduce a linear correlation coefficient as a measure for studying the linear dependency between the slowlyvarying complex forcing amplitudes for the linear and vibro-impact beams and demonstrate that only a set of lower-frequency modes are strongly influenced by vibro-impacts, capturing most of the essential nonlinear dynamics. These results demonstrate the efficacy of the proposed approach to analyze strongly nonlinear measured time series and provide physical insight for strong nonlinear dynamical interactions.

Keywords Nonlinear system identification · Empirical mode decomposition · Vibro-impact beam · Intrinsic mode oscillation · Nonlinear interaction model

$\begin{array}{ll}\text { Abbreviations } \\ \text { DOF } & \text { Degree-of-freedom } \\ \text { EMD } & \text { Empirical mode decomposition } \\ \text { FEP } & \text { Frequency-energy plot } \\ \text { FT } & \text { Fourier transform } \\ \text { HT } & \text { Hilbert transform } \\ \text { IMF } & \text { Intrinsic mode function } \\ \text { IMO } & \text { Intrinsic modal oscillator } \\ \text { NIM } & \text { Nonlinear interaction model } \\ \text { NSI } & \text { Nonlinear system identification } \\ \text { POD } & \text { Proper orthogonal decomposition } \\ \text { ROM } & \text { Reduced-order model } \\ \text { VI } & \text { Vibro-impact }\end{array}$

\section{Introduction}

Experimental modal analysis based on Fourier transforms (FTs) has been well established based on the assumption of linearity and stationarity of the measured signals (see, for example, [1]). In many practical situations, however, the measured data is likely to exhibit strong nonlinearity and nonstationarity, particularly when the tested systems involve nonlinearities due to complexity caused by multi-physics nonlinear interactions [2]. In addition, FT-based methods are not able to properly isolate and extract nonlinearity and nonstationarity from the measured data, frequently leading to wrong conclusions (for example, to misinterpretations of internal and combination resonances as natural frequencies). As a result, there has been the need for an effective, straightforward, system identification and reduced-order modeling method for characterizing strongly nonlinear and nonstationary, complex, multi-component systems in multi-physics applications.

Reviews of nonlinear system identification (NSI) and reduced-order modeling (ROM) methods are provided in $[3,4]$. Typical nonparametric NSI methods include proper orthogonal decomposition (POD, also known as Karhunen-Loève decomposition [5-8]), smooth orthogonal decomposition [9], Volterra theory [10,11], Kalman filter [12], and so on. As for the methods of nonlinear parameter estimation, we mention the restoring force surface method [13], NARMAX (Nonlinear Auto-Regressive Moving Average models with eXogenous inputs) methods [14], harmonic balance method [15], methods based on Hilbert transform [16,17], and others.

Use of POD has been rather popular in studying system identification and nonlinear normal modes of coupled beams [18] and rods [19], and in structural damage detection [20]. For example, the method of POD has been utilized for studying chaotic vibrations of a 10-degree-of-freedom (DOF) impact oscillator and a flexible-beam impact oscillator in [21] and [22], respectively. In these studies, the spatial structure of impacting responses under a harmonic excitation of the boundary was demonstrated to be close to what can be obtained by averaging over many impulse-response tests on the linear system (even if the system is strongly nonlinear). Moreover, POD was applied for model reduction of a vibro-impact (VI) rod [23] and also for extracting dominant coherent structures of a VI beam from experimental time series data [24] with the goal to eventually derive low-dimensional ROMs through a Galerkin reconstruction process based on the extracted mode shape functions.

We note, however, that these techniques are only applicable to specific classes of dynamical systems; in addition, some functional form is assumed for modeling the system nonlinearity. Recently, a nonlinear system identification (NSI) method with the promise of broad applicability was proposed by Lee et al. [25]. This 

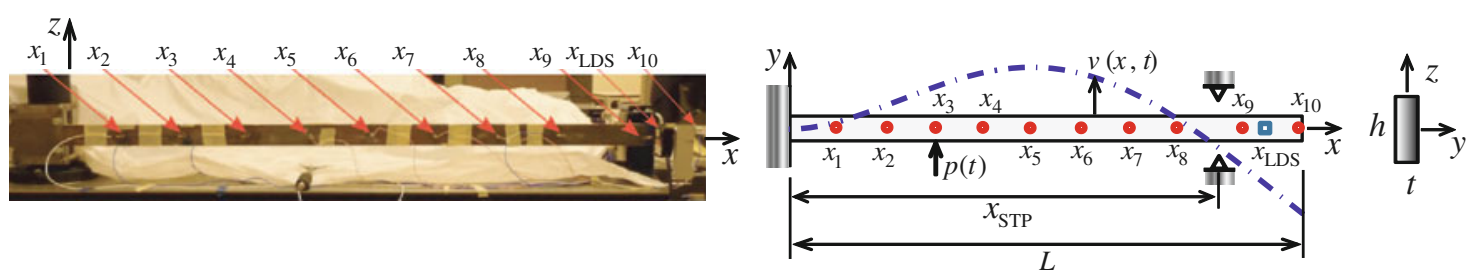

Fig. 1 Experimental setup for the VI beam: $x_{1}-x_{10}, x_{\text {LDS }}$, and $x_{\text {STP }}$ respectively denote the spanwise locations of the accelerometers, of the laser displacement sensors, and of the rigid stops

Table 1 Positions of the accelerometers, rigid stops, and laser displacement sensors of the VI beam

\begin{tabular}{lllllllllllll}
\hline & $x_{1}$ & $x_{2}$ & $x_{3}$ & $x_{4}$ & $x_{5}$ & $x_{6}$ & $x_{7}$ & $x_{8}$ & $x_{9}$ & $x_{10}$ & $x_{\text {STP }}$ & $x_{\text {LDS }}$ \\
\hline Positions $(\mathrm{mm})$ & 131 & 263 & 395 & 527 & 657 & 787 & 917 & 1,052 & 1,215 & 1,311 & 1,185 & 1,230 \\
\hline
\end{tabular}

method was based on empirical mode decomposition (EMD [26]), under the key assumption that the measured time series can be decomposed in terms of a finite number of oscillating components. These are in the form of fast (nearly) monochromatic oscillations modulated by slowly varying amplitudes. The empirical slow-flow model of the dynamics is obtained from EMD, and its correspondence with the analytical slow-flow model has been established [27], paving the way for constructing physics-based local nonlinear interaction models (NIMs) [28].

A NIM consists of a set of intrinsic modal oscillators (IMOs) that can reproduce the measured time series over different time scales and account for (even strongly) nonlinear modal interactions across scales. Hence, it represents a local model of the dynamics, identifying specific nonlinear transitions. By collecting energydependent frequency behaviors from all identified IMOs, a frequency-energy plot can be constructed, which depicts global features of the dynamical system. The method requires no a priori system information but only measured (or simulated) time series; that is, it is purely an output-based approach. Applications of the proposed NSI methodology have been provided with studies of targeted energy transfers in a 2-DOF dynamical system [28], instability generation and suppression in a 2-DOF rigid aeroelastic wing model [29], and the dynamics of a rod coupled to an essentially nonlinear end attachment [30].

In this paper, we explore the nonlinear dynamics of a VI beam (whose setup is similar to that used in [24]) by performing the aforementioned NSI method [25] to reveal coherent structures (e.g., Dawes [31]) in terms of IMOs of strongly nonlinear dynamics due to vibro-impacts. Study of such systems will provide essential dynamical features of structures with defects with applications to structural health monitoring and damage detection (e.g., [32,33]). For this purpose, this paper has the following structure. Section 2 provides a discussion of the VI beam model including geometry, measurement locations, method of excitation, (linearized) natural frequencies and mode shapes; in Sect. 3, the proposed NSI method is applied to the numerically obtained acceleration data of the VI beam and those of the underlying linearized beam for comparison purposes; then, some of the experimental results are compared with the corresponding numerical data in Sect. 4, and concluding remarks are provided in Sect. 5.

\section{System descriptions}

We consider the uniform, homogeneous cantilever beam (made of steel with the density $\rho=7,850 \mathrm{~kg} / \mathrm{m}^{3}$ and Young's modulus $E=200 \mathrm{GPa}$ ) depicted in Fig. 1, with dimensions $L \times h \times t=1.311 \times 0.0446 \times 0.008 \mathrm{~m}$ so that the cross-sectional area and the second moment of area with respect to the $z$ axis are $A=3.57 \times 10^{-4} \mathrm{~m}^{2}$ and $I_{z z}=1.9 \times 10^{-9} \mathrm{~m}^{4}$, respectively (we refer to Fig. 1 for a definition of the system of axes). Table 1 summarizes the positions of the accelerometers $x_{1}-x_{10}$ along the beam span, the position of the laser displacement sensor $x_{\text {LDS }}$, and the placement of the two symmetric rigid stops $x_{\text {STP }}$ causing vibro-impacts. The leading ten natural frequencies (theoretical and experimental) $\omega_{n}$ in $\mathrm{Hz}$ are listed in Table 2, with the corresponding normalized mode shape functions $\phi_{n}(x / L), n=1, \ldots, 10$, being presented in Fig. $2[34,35]$.

Two clearance levels between the cantilever beam and the rigid stops are considered, namely, infinite clearance corresponding to the case of the linear cantilever beam, and $4 \mathrm{~mm}$ clearance corresponding to the case of the strongly nonlinear VI beam. Experimental procedures for measuring time series data involve (i) applying impulsive excitation $p(t)$ by varying magnitude at position $x_{3}$ by means of an impact hammer, 
Table 2 The leading 10 linear natural frequencies (in Hz) of the linear cantilever beam in Fig. 1 (cf. [34,35])

\begin{tabular}{|c|c|c|c|c|c|c|c|c|c|c|}
\hline$\omega_{n}$ & $1 \mathrm{st}$ & 2nd & $3 \mathrm{rd}$ & 4th & 5 th & 6th & 7th & 8th & 9th & 10th \\
\hline Theoretical & 3.8 & 23.8 & 66.6 & 130.5 & 215.7 & 322.2 & 450.0 & 599.1 & 769.5 & 961.2 \\
\hline Experimental & 3.7 & 23.2 & 64.9 & 126.9 & 209.4 & 314.7 & 433.9 & 580.7 & 751.3 & 926.7 \\
\hline
\end{tabular}

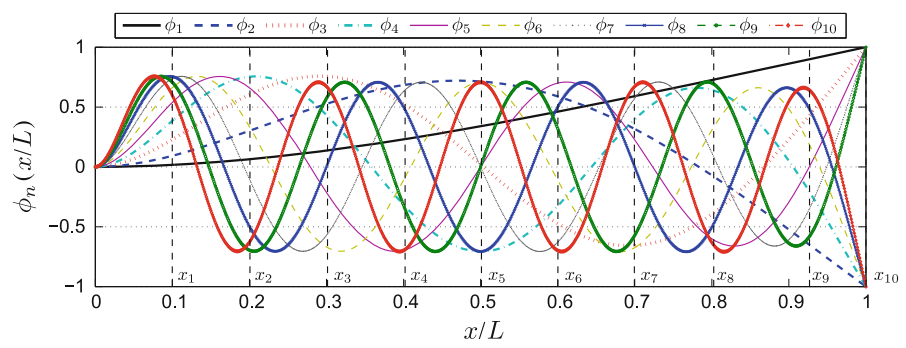

Fig. 2 The normalized mode shape functions $\phi_{n}(x / L)$ of a cantilever beam: The first ten modes are depicted, and the vertical dashed lines denote the positions for accelerometers

selecting the excitation frequency band by using several types of tips on the impact hammer (e.g., plastic, rubber, and metal), and (ii) measuring the resulting accelerations at $x_{1}$ through $x_{10}$ and the displacement at $x_{\text {LDS }}$.

In this work, we utilize numerically generated acceleration signals from a reduced-order model based on the assumed-modes method, and such numerical solutions are updated and validated by the experimental measurements. That is, the beam was excited at each node with an impact hammer, and averages of four measurements were taken at each node; from the resulting 100 transfer functions, the leading 10 mode shapes, modal damping factors, and natural frequencies were obtained and used to update the assumed-modes model. In the assumed-modes method, the analytical natural frequencies were replaced with the measured ones, and numerically simulated time series were obtained by solving the reduced system of differential equations. Details of this computation can be found in [34].

We remark that the 5th mode, whose linearized natural frequency is equal to $209 \mathrm{~Hz}$, has a node at $x_{9}$, which is located very close to the point of vibro-impacts $x_{\mathrm{STP}}$. Furthermore, the impulsive excitation is applied at location $x_{3}$, which is also close to another node of the 5th mode. As shown below, this will affect the results of EMD analysis used for reconstructing the 5th mode at those particular points (i.e., there will arise issues of observability) in the sense that the flexible dynamics of the beam at these locations is small and consequently is dominated by the vibro-impacts (non-smooth effects). Similar observations apply for the 8th mode, which possesses a node near the excitation point $\left(x_{3}\right)$.

\section{Nonlinear system identification (NSI) of the vibro-impact beam}

In this section, we apply the NSI methodology to two typical cases: (i) The linear beam (i.e., a cantilever beam with infinite clearances at the impact boundaries); and (ii) the vibro-impact (VI) beam with $4 \mathrm{~mm}$ symmetric clearances. In particular, by comparing the system identification results for the VI beam to those of the linear beam, we wish to study the effects of the strongly nonlinear dynamics induced by the vibro-impacts. Typically, we will consider the acceleration signals at position $x_{9}$ for this comparison because the VI effects are expected to be most significant there due to its proximity to the impact position. We also wish to illustrate overall spatio-temporal variations of the beam dynamics caused by vibro-impacts. First, we briefly discuss the basic elements of the NSI methodology.

\subsection{NSI methodology}

Once the (numerical) simulation data are computed, the first step of the proposed NSI methodology is to perform empirical mode decomposition (EMD) [26,27] on the simulated time series, which yields a complete and nearly (but not thoroughly) orthogonal basis of intrinsic mode functions (IMFs) at each sensing location. These are oscillatory modes embedded in the time series, each with its own characteristic time scale, whose 
linear superposition reconstructs the measured time series. Hence, EMD provides a multi-scale decomposition of a measured time series in terms of embedded oscillatory modes in the measured data at different time scales of the dynamics. Although EMD was originally conceived as an ad hoc decomposition method, as discussed below it can be provided with a physics-based foundation. First, we discuss the basic computational elements of this decomposition.

The main loop of the EMD algorithm [26] for extracting the IMFs from a signal $x(t)$ consists of the following steps: (i) identify all extrema of $x(t)$; (ii) perform (spline) interpolations between minima (maxima), resulting in an envelope $e_{\min }(t)\left(e_{\max }(t)\right)$; (iii) compute the average $R(t)=\left[e_{\min }(t)+e_{\max }(t)\right] / 2$ (considered as a residual); (iv) extract the remainder $c(t)=x(t)-R(t)$; (v) iterate on the residual $R(t)$. In practice, the above procedure is refined by a sifting process, and the inner loop that iterates (i)-(iv) on the detail $c(t)$ runs until the average $R(t)$ can be considered zero-mean under some tolerance (i.e., as a stopping criterion). Once achieved, the detail $c(t)$ is regarded as the effective IMF.

The NSI methodology is based on the basic assumption that the measured dynamics contains $N$ distinct dominant components at frequencies, $\omega_{1}, \omega_{2}, \ldots, \omega_{N}$, which are well separated and follow the ordering $\omega_{1}<\omega_{2}<\cdots<\omega_{N}$. Then, by applying the EMD, the measured signal is decomposed in terms of $N$ dominant IMFs (corresponding to the $N$ dominant harmonic components in the signal), so the response $x(t)$ can be expressed as

$$
x(t) \approx c_{1}(t)+c_{2}(t)+\cdots+c_{N}(t)
$$

where $c_{m}(t), m=1,2, \ldots, N$, indicates the $m$ th dominant IMF associated with the fast frequency $\omega_{m}$. We remark that, by construction, each IMF is a function that possesses zero local mean with one zero between any two consecutive local extrema, and, also by construction, is a narrowband signal. The dominant IMFs are determined by performing Hilbert transform (HT) of the computed IMFs in order to extract their instantaneous frequencies and comparing them to wavelet transform spectra of the corresponding time series. This procedure determines the dominant IMFs and the corresponding fast frequencies in the dynamics at each sensing location and also identifies the basic time scales and the dimensionality of the measured dynamics. Furthermore, motivated by the fact that a complex function whose imaginary part is the HT of the real part is analytic, we can complexify the $m$ th $\operatorname{IMF} c_{m}(t)$ by defining the following analytic complex function

$$
\hat{\psi}_{m}(t)=c_{m}(t)+j \mathcal{H}\left[c_{m}(t)\right] \triangleq \hat{A}_{m}(t) \mathrm{e}^{j \hat{\theta}_{m}(t)} \mathrm{e}^{j \omega_{m} t}, \quad j^{2}=-1
$$

where $\mathcal{H}[\cdot]$ denotes Hilbert transform. The slowly-varying envelope and phase of the $m$ th IMF are computed as $\hat{A}_{m}(t)=\sqrt{c_{m}(t)^{2}+\mathcal{H}\left[c_{m}(t)\right]^{2}}$ and $\hat{\theta}_{m}(t)=\tan ^{-1}\left[\mathcal{H}\left[c_{m}(t)\right] / c_{m}(t)\right]-\omega_{m} t$, respectively.

As mentioned previously, the EMD procedure is performed in an ad hoc fashion. As shown in previous works [27,28], however, a physics-based foundation for EMD can be provided based on the slow flow of the underlying dynamics. The analytical slow-flow dynamics (assuming that the equations of motion are known) is studied using the complexification-averaging technique [36]. To this end, we express the response $x(t)$ in Eq. (1) as the sum of $N$ independent components,

$$
x(t)=x_{1}(t)+x_{2}(t)+\cdots+x_{N}(t)
$$

where the subscripts follow the same convention as in Eq. (1). For each component of Eq. (3), we assign a new complex variable defined by

$$
\psi_{m}(t)=\dot{x}_{m}(t)+j \omega_{m} x_{m}(t) \triangleq \varphi_{m}(t) \mathrm{e}^{j \omega_{m} t}, \quad j^{2}=-1
$$

where a slow/fast partition of the dynamics in terms of the slow (complex) amplitude $\varphi_{m}(t)$ and the fast oscillation $\mathrm{e}^{j \omega_{m} t}$ was assumed. Substituting into the equations of motion and performing multiphase averaging [37] for each of the fast frequencies, we obtain the analytical slow flow of the dynamical system.

Noting that complexifying the identified IMFs in Eq. (2) as $\hat{\psi}_{m}(t)$ results in the same form as the complexification (4) in the slow-flow construction, we have a direct way to relate the IMFs to the governing slow-flow dynamics, in particular to the complex amplitudes, $\psi_{m}(t)$. This provides a way to physically interpret the (previously ad hoc) dominant IMFs in terms of the slow-flow dynamics. Moreover, these partitions can be directly related, since they represent identical theoretical and numerical multi-scale slow-fast decompositions of the measured time series, that is, $x_{m}(t)$ from the theoretical model and $c_{m}(t)$ from the numerical model.

$$
x_{m}(t) \Leftrightarrow c_{m}(t), \quad m=1, \ldots, N
$$


Now, we introduce the alternative complexification of IMFs that resembles that of the analytical complexification (4),

$$
\check{\psi}_{m}(t)=\dot{c}_{m}(t)+j \omega_{m} c_{m}(t) \triangleq \check{A}_{m}(t) \mathrm{e}^{j \check{\theta}_{m}(t)} \mathrm{e}^{j \omega_{m} t}, \quad j^{2}=-1
$$

where $\check{A}_{m}(t)=\sqrt{\dot{c}_{m}(t)^{2}+\omega_{m}^{2} c_{m}(t)^{2}}$ and $\check{\theta}_{m}(t)=\tan ^{-1}\left[\omega_{m} c_{m}(t) / \dot{c}_{m}(t)\right]-\omega_{m} t$. Simple manipulations [28] prove that the alternative complexifications of the IMFs (2) and (6) are related through

$$
\hat{\psi}_{m}(t)=\frac{1}{j \omega_{m}} \check{\psi}_{m}(t)
$$

which suggests that the complexifications must be of the form

$$
\left[\psi_{m}(t) \Leftrightarrow \check{\psi}_{m}(t)=j \omega_{m} \hat{\psi}_{m}(t)\right] \Longrightarrow\left[\varphi_{m}(t) \Leftrightarrow j \omega_{m} \hat{A}_{m}(t) \mathrm{e}^{j \hat{\theta}_{m}(t)}\right]
$$

Relations (8) provide a physics-based theoretical foundation for EMD, whereby the dominant IMFs represent the underlying slow flow of the dynamics and, hence, capture all the important (multi-scale) dynamics. This leads us to the important conclusion that dominant IMFs extracted by means of EMD of experimentally measured time series (i.e., from systems whose governing equations are not known) correspond to the underlying slow-flow dynamics of the tested dynamical system.

Based on the correspondence (8) between the theoretical and empirical slow-flow analyses, we address the local aspect of the NSI methodology by constructing a reduced-order model (ROM) or, more generally, a nonlinear interaction model (NIM) in terms of intrinsic modal oscillators (IMOs). We define the IMOs as the equivalent set of linear oscillators that can reproduce a measured time series at different time scales. IMOs are typically expressed as sets of linear, damped oscillators having as forcing functions the nonlinear modal interactions [28]. A basic requirement is that each IMO should approximately reproduce the corresponding dominant IMF (i.e., the one corresponding to the same fast frequency), provided that the fast frequencies of the time series are well separated (distinct). For the $m$ th component of $x(t)$ in Eq. (3), we write

$$
\ddot{x}_{m}(t)+2 \hat{\zeta}_{m} \omega_{m} \dot{x}_{m}(t)+\omega_{m}^{2} x_{m}(t)=F_{m}(t)
$$

where the coefficients $\hat{\zeta}_{m}$ and $\omega_{m}$ are assumed to be constant, and the nonhomogeneous term $F_{m}(t)$ represents a time-dependent forcing term describing the nonlinear modal interaction of the $m$ th component of $x(t)$ with the other components of the measured time series. Equivalently, we assume that each of the components in Eq. (3) is generated by an IMO of the form (9). In principle, the nonhomogeneous term in (9) can be expressed in terms of slow/fast partitions of all participating frequency components. That is, we can write,

$$
F_{m}(t)=\operatorname{Re}\left[\Lambda_{1}(t) \mathrm{e}^{j \omega_{1} t}+\Lambda_{2}(t) \mathrm{e}^{j \omega_{2} t}+\cdots+\Lambda_{m}(t) \mathrm{e}^{j \omega_{m} t}+\cdots+\Lambda_{N}(t) e^{j \omega_{N} t}\right]
$$

where $\operatorname{Re}[\cdot]$ represents the real part, and $\Lambda_{m}(t)$, the slowly-varying (complex) forcing amplitude. However, because of the linear structure of the IMO, it should be clear that the only term that can produce an $\mathcal{O}(1)$ dynamic response is the one with fast frequency $\omega_{m}$ (i.e., the eigenfrequency of the IMO). Hence, it is justified to approximately express the IMO in the simplified form

$$
\ddot{x}_{m}(t)+2 \hat{\zeta}_{m} \omega_{m} \dot{x}_{m}(t)+\omega_{m}^{2} x_{m}(t) \approx \operatorname{Re}\left[\Lambda_{m}(t) \mathrm{e}^{j \omega_{m} t}\right]
$$

where $m=1, \ldots, N$ and the nonhomogeneous term is in the form of a slowly modulated oscillation with fast frequency equal to the eigenfrequency of the IMO.

The issue is now to identify the modal parameters of the IMO (11) and, more importantly, its forcing term representing the nonlinear modal interaction at the respective time scale (frequency). To this end, we apply complexification-averaging technique by introducing the new complex variable (4), which, when substituted into Eq. (11), yields the complexification of the IMO

$$
\begin{aligned}
& {\left[\dot{\varphi}_{m}(t)+j \omega_{m} \varphi_{m}(t)\right] \mathrm{e}^{j \omega_{m} t}-\frac{j \omega_{m}}{2}\left[\varphi_{m}(t) \mathrm{e}^{j \omega_{m} t}+\varphi_{m}(t)^{*} \mathrm{e}^{-j \omega_{m} t}\right]} \\
& \quad+2 \frac{\zeta_{m} \omega_{m}}{2}\left[\varphi_{m}(t) \mathrm{e}^{j \omega_{m} t}+\varphi_{m}(t)^{*} \mathrm{e}^{-j \omega_{m} t}\right]+\frac{\omega_{m}^{2}}{2 j \omega_{m}}\left[\varphi_{m}(t) \mathrm{e}^{j \omega_{m} t}-\varphi_{m}(t)^{*} \mathrm{e}^{-j \omega_{m} t}\right] \\
& \approx \frac{1}{2}\left[\Lambda_{m}(t) \mathrm{e}^{j \omega_{m} t}+\Lambda_{m}{ }^{*}(t) \mathrm{e}^{-j \omega_{m} t}\right]
\end{aligned}
$$


Multiplying both sides of Eq. (12) by $\mathrm{e}^{-j \omega_{m} t}$ and averaging out fast terms other than $\mathrm{e}^{j \omega_{m} t}$, we derive a relation between the forcing amplitude of the nonlinear modal interaction $\Lambda_{m}(t)$ and the complex amplitude $\varphi_{m}(t)$ as follows,

$$
\Lambda_{m}(t) \approx 2\left[\dot{\varphi}_{m}(t)+\hat{\zeta}_{m} \omega_{m} \varphi_{m}(t)\right] \approx 2\left[\frac{\mathrm{d}}{\mathrm{d} t}\left(j \omega_{m} \hat{A}_{m}(t) \mathrm{e}^{j \hat{\theta}_{m}(t)}\right)+j \hat{\zeta}_{m} \omega_{m}^{2} \hat{A}_{m}(t) \mathrm{e}^{j \hat{\theta}_{m}(t)}\right]
$$

where relation (8) was taken into account. This provides a way to estimate the nonlinear modal interaction force provided that the eigenfrequency and viscous damping ratio of the IMO are known. The eigenfrequency $\omega_{m}$ is directly determined by performing wavelet analysis of the time series and constructing wavelet transform spectra in time [27]. The viscous damping factor $\hat{\zeta}_{m}$ is determined through an optimization process based on the requirement that the response of the IMO should reproduce the IMF corresponding to the dominant frequency $\omega_{m}$; hence, the damping factor is selected by minimizing the normalized mean square errors between the envelope of the IMF $c_{m}(t)$ and the IMO solution $x_{m}(t)$ [28].

As discussed in [25], following these steps local models of the dynamics are constructed, modeling specific nonlinear damped transitions of the system under specific applied excitations. Global models of the dynamics can then be synthesized from the local models by depicting the local models in a frequency-energy plot (FEP) at different frequency and energy ranges. This is performed by wavelet transforming individual time series and depicting the resulting spectra in the frequency-energy domain. Since the global aspect of the NSI methodology will not be considered herein, we will not elaborate any further on the construction of the global FEP models, instead referring to [25].

\subsection{Linear beam}

By linear beam, we mean the cantilever beam in Fig. 1 without any rigid stops (or with impacting boundaries of infinite clearances). Then, since the beam is homogeneous and uniform, we can assume that its transverse vibrations can be approximately governed by the Bernoulli-Euler beam model with the following equation of motion,

$$
\rho A \ddot{v}(x, t)+E I_{z z} v^{\prime \prime \prime \prime}(x, t)=p(t) \delta\left(x-x_{3}\right)
$$

where $v(x, t)$ denotes the displacement of the beam in the transverse $(y)$ direction at $(x, t)$ (cf. Fig. 1); $p(t)=P_{0} \delta(t)$ is the impulsive excitation at $t=0$, where $\delta(t)$ and $\delta(x)$ denote Dirac delta functions; and primes and dots are partial differentiation with respect to $x$ and $t$, respectively. Then, the general solution for Eq. (14) can be written as

$$
v(x, t)=\sum_{m=1}^{\infty} A_{m} \phi_{m}(x) \mathrm{e}^{-\zeta_{m} \omega_{m} t} \cos \left(\omega_{m d} t-\theta_{m}\right)
$$

where $\omega_{m d}=\omega_{m} \sqrt{1-\zeta_{m}^{2}} ; \omega_{m}$ is the natural frequency of the $m$ th linear bending mode; $\zeta_{m}$ is the modal damping factor (when a certain viscous damping is assumed in the system); and $\phi_{m}(x)$ is the normalized mode shape function for the $m$ th mode (cf. Fig. 2). The corresponding acceleration can be written as

$$
a(x, t) \triangleq \ddot{v}(x, t)=\sum_{m=1}^{\infty} \bar{A}_{m} \phi_{m}(x) \mathrm{e}^{-\zeta_{m} \omega_{m} t} \cos \left(\omega_{m d} t-\bar{\theta}_{m}\right)
$$

where $\bar{A}_{m}=A_{m} \omega_{m}^{2}$ and $\bar{\theta}_{m}=\theta_{m}+\tan ^{-1}\left[2 \zeta_{m} \sqrt{1-\zeta_{m}^{2}} /\left(1-2 \zeta_{m}^{2}\right)\right]$.

Consider now that the acceleration response of the linear beam at position $x_{9}$ depicted in Fig. 3. The wavelet and Fourier transforms clearly depict the ten dominant fast frequencies identified from experimental modal analysis (see Table 2). As discussed in Sect. 2, the harmonic at $209 \mathrm{~Hz}$ appears to be negligible because the position $x_{9}$ is close to one of the nodes for the 5 th mode. We write

$$
a_{9}(t) \equiv a\left(x_{9}, t\right) \approx \sum_{m=1}^{10} \bar{A}_{m} \phi_{m}\left(x_{9}\right) \mathrm{e}^{-\zeta_{m} \omega_{m} t} \cos \left(\omega_{m} t-\bar{\theta}_{m}\right)
$$




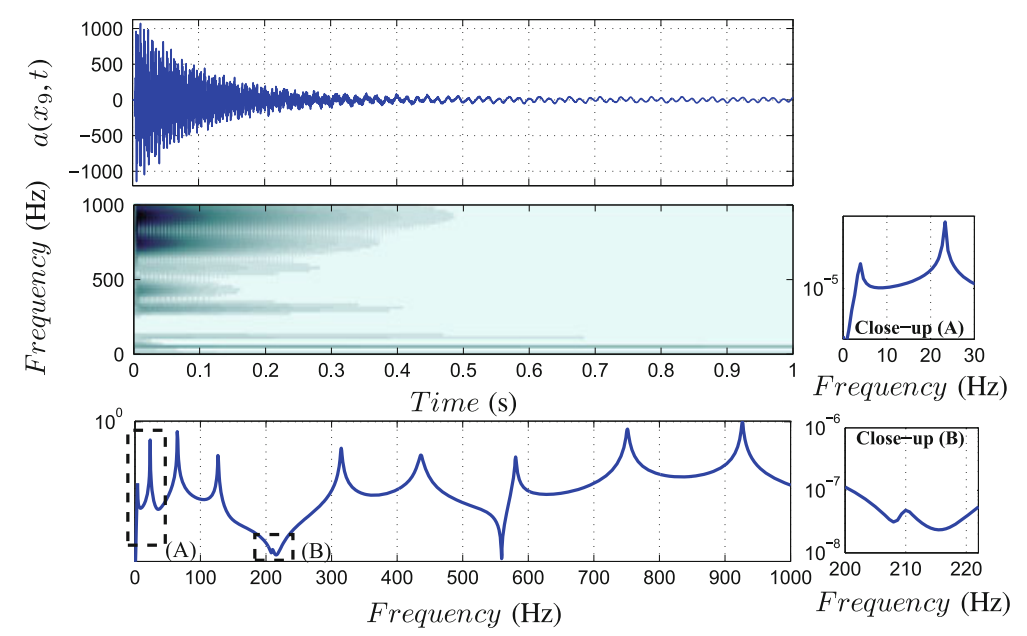

Fig. 3 Wavelet and Fourier transforms of the acceleration for the linear beam at position $x_{9}$

for small damping $\zeta_{m}$. By means of EMD analysis, we wish to obtain the relation between the acceleration time series and the intrinsic mode functions (IMFs) such that

$$
a_{9}(t) \approx \sum_{m=1}^{10} c_{m}\left(a_{9}, t\right) \approx \sum_{m=1}^{10} \bar{A}_{m} \phi_{m}\left(x_{9}\right) \mathrm{e}^{-\zeta_{m} \omega_{m} t} \cos \left(\omega_{m} t-\bar{\theta}_{m}\right)
$$

where $c_{m}\left(a_{9}, t\right)$ denotes the $m$ th IMF of the acceleration at position $x_{9}$ (and is usually associated with the $m$ th normal mode vibration that can be observed at the same position of the beam). Figure 4 depicts the ten dominant IMFs from the advanced EMD analysis algorithm introduced in [27], demonstrating that the relation (18) is valid except for the 5th mode of the beam due to observability issues. (For this kind of a linear response, one might get similar or even better decomposition with a typical bandwidth filter.)

We establish the reduced-order model (ROM) for the acceleration in Fig. 3 for the linear beam dynamics at position $x_{9}$ in terms of intrinsic modal oscillators (IMOs). That is, we write the IMO corresponding to each IMF as in Eq. (11), and the instantaneous slowly-varying envelope and phase of the $m$ th IMF are computed respectively as

$$
\hat{A}_{m}(t)=\sqrt{c_{m}\left(a_{9}, t\right)^{2}+\mathcal{H}\left[c_{m}\left(a_{9}, t\right)\right]^{2}}, \quad \hat{\theta}_{m}(t)=\tan ^{-1}\left\{\mathcal{H}\left[c_{m}\left(a_{9}, t\right)\right] / c_{m}\left(a_{9}, t\right)\right\}-\omega_{m} t
$$

where $m=1, \ldots, 10$. Since the slowly-varying complex forcing amplitude $\Lambda_{m}(t)$ is computed from the time series (or IMF) in an effort to match the solution $x_{m}(t)$ of the IMO with the corresponding IMF [cf. Eq. (13)], we can write $x_{m}(t) \approx c_{m}\left(a_{9}, t\right)$. During this validation process, the damping factor $0<\hat{\zeta}_{m}<1$ is chosen such as to minimize the error between $x_{m}(t)$ and $c_{m}\left(a_{9}, t\right)$. Then, the original response can be reconstructed as the sum of all IMO solutions; that is, the following expression holds:

$$
a_{9}(t) \approx \sum_{m=1}^{10} x_{m}(t) \approx \sum_{m=1}^{10} c_{m}\left(a_{9}, t\right)
$$

Figure 5 compares the 10th and 5th IMFs with the corresponding IMO solutions, exhibiting good agreement.

Finally, we consider the physical meaning of the complex-valued forcing function $\Lambda_{m}(t)$ for the $m$ th IMO of the linear problem, since such a term is known to be associated with nonlinear modal interactions in nonlinear dynamical systems $[28,29]$. In our linear beam problem, the slowly-varying envelope $\hat{A}_{m}(t)$ and phase $\hat{\theta}_{m}(t)$ in Eq. (8) can be identified from Eq. (18) as

$$
\hat{A}_{m}(t)=\bar{A}_{m} \phi_{m}\left(x_{9}\right) \mathrm{e}^{-\zeta_{m} \omega_{m} t}, \quad \hat{\theta}_{m}(t)=-\bar{\theta}_{m}=\text { constant }
$$

Then, the slow-flow variable in Eq. (8) can be expressed as

$$
\varphi_{m}(t) \approx j \omega_{m} \bar{A}_{m} \phi_{m}\left(x_{9}\right) \mathrm{e}^{-j \bar{\theta}_{m}} \mathrm{e}^{-\zeta_{m} \omega_{m} t} \Rightarrow \dot{\varphi}_{m}(t) \approx-j \zeta_{m} \omega_{m}^{2} \bar{A}_{m} \phi_{m}\left(x_{9}\right) \mathrm{e}^{-j \bar{\theta}_{m}} \mathrm{e}^{-\zeta_{m} \omega_{m} t}
$$



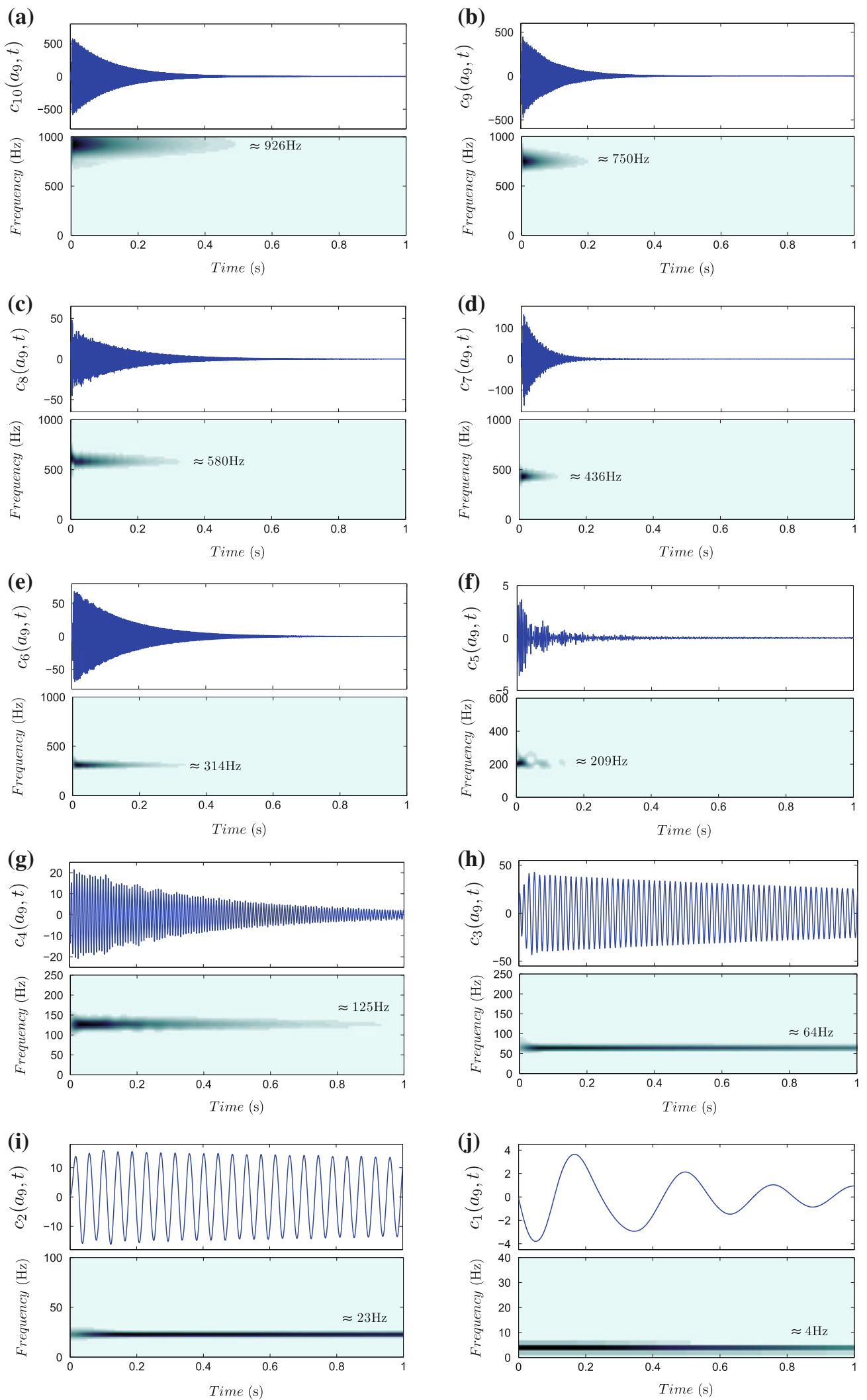

Fig. 4 The 10 dominant IMFs extracted from the acceleration response in Fig. 3: a through $\mathbf{j}$ sequentially depict the 10th to 1st IMFs, respectively 
(a)

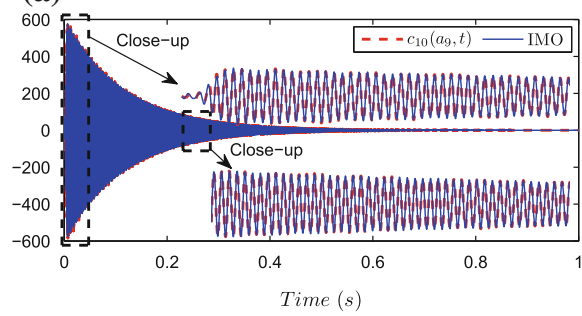

(b)

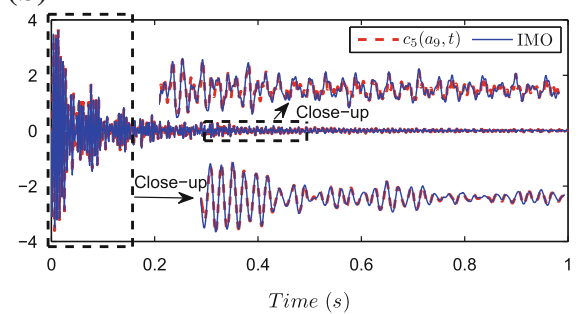

Fig. 5 Comparison of the IMFs in Fig. 4 with their corresponding IMO solutions: a 10th IMF; b 5th IMF

If $\hat{\zeta}_{m}=\zeta_{m}$ (i.e., the damping factor in the IMO is the same as the modal damping factor identified from experimental modal analysis, and carries a direct physical meaning), then we can easily show that $\Lambda_{m}(t) \approx 0$ due to Eq. (13). This idea may sound feasible and reasonable, because the resulting reduced-order model will be the same as that obtained from the typical linear modal analysis with the coordinates, $x_{m}, m=1, \ldots, 10$, being the modal coordinates. Furthermore, the solution for the IMO (11) will appear as a free damped response, which may naturally satisfy the relation in Eq. (18). However, as is the case for many other nonlinear system identification methods where it is of more interest to check whether the proposed parametric model is able to reproduce the measured (or simulated) dynamics, the damping factor in the IMO is not necessarily the same as the physical one (i.e., $\hat{\zeta}_{m} \neq \zeta_{m}$, in general). In this case, the complex forcing amplitude $\Lambda_{m}(t)$ can be expressed as

$$
\Lambda_{m}(t) \approx j\left(\hat{\zeta}_{m}-\zeta_{m}\right) \omega_{m}^{2} \bar{A}_{m} \phi_{m}\left(x_{9}\right) \mathrm{e}^{-j \bar{\theta}_{m}} \mathrm{e}^{-\zeta_{m} \omega_{m} t}
$$

The absolute value of the complex number (23) is a monotonically and exponentially decaying function (cf. Fig. 11 in Sect. 3.3); such forcing function will not generate any modal interactions (as is supposed to be the case for a linear system). Nonetheless, the solution for the IMO (11), which is strongly driven by the forcing $\Lambda_{m}(t) \mathrm{e}^{j \omega_{m} t}$ because $\hat{\zeta}_{m} \gg \zeta_{m}$, can approximately reproduce the IMF in Eq. (18). Similar discussions can be made not only for the response at position $x_{9}$, but also for those at all other positions along the linear beam.

\subsection{Vibro-impact (VI) beam}

We now consider the cantilever beam in Fig. 1 but with the two symmetric rigid stops of $4 \mathrm{~mm}$ clearances at position $x_{\mathrm{STP}}$. If the displacement $\left|v\left(x_{\mathrm{STP}}, t\right)\right|<4 \mathrm{~mm}$, then the dynamics of the beam is linear and can be described by Eq. (14). Whenever the beam displacement $\left|v\left(x_{\mathrm{STP}}, t\right)\right|=4 \mathrm{~mm}$, a vibro-impact occurs resulting in a new impact load $\bar{p}(t) \delta\left(x-x_{\mathrm{STP}}\right)$ applied to the beam as well as causing energy dissipation due to inelastic impact. Mathematically speaking, the nonsmoothness due to the vibro-impacts means that the displacement response is of class $C^{0}$ (i.e., continuous but not continuously differentiable).

For this strongly nonlinear nonsmooth dynamical system, there is no closed-form solution available, in general. Furthermore, such a VI dynamical system may possess a very complicated topological structure of periodic orbits (e.g., see [38]). This is mainly because nonsmooth dynamical systems may involve complicated dynamics such as grazing bifurcations [39] and chaos [22]. We wish to model and understand the nonlinear dynamics of the VI beam by applying the proposed NSI method.

As for the case of the linear beam problem of the previous section, we consider the acceleration signal at position $x_{9}$ (depicted in Fig. 6), where the effects of vibro-impacts generate multiple broadband perturbations in the wavelet transforms. In particular, comparing the Fourier transform of the linear beam response (dashed line) with that of the VI beam, this broadband excitation of the beam due to vibro-impacts is significant. Figure 7a depicts the numerically computed displacement and the corresponding impact load on the beam at $x_{\text {STP }}$ in order to identify the instants of vibro-impacts (i.e., the time instants when the beam displacement at $x_{\text {STP }}$ reaches the thresholds $\pm 4 \mathrm{~mm}$ ).

It was shown in [38] that the nonlinear modal interactions due to vibro-impacts are purely due to the smooth parts of the VI dynamics, whereas the nonsmooth parts tend to create frequency-energy relations involving numerical artifacts. Such numerical artifacts could lead to wrong conclusions regarding the nonlinear resonances involved in the nonlinear modal interactions between the measured IMFs. Furthermore, it was demonstrated that the smooth parts of the VI dynamics can be obtained by separating the nonsmooth 

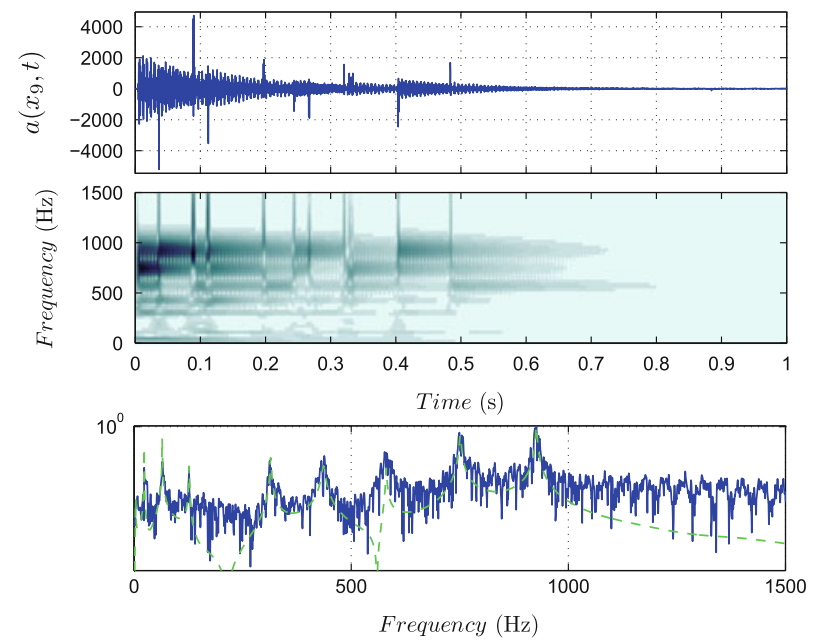

Fig. 6 Wavelet and Fourier transforms of the acceleration for the VI beam at $x=x_{9}$ (The Fourier transform in Fig. 3 is superimposed as a dashed line to illustrate the effects of vibro-impacts in frequency domain)

(a)

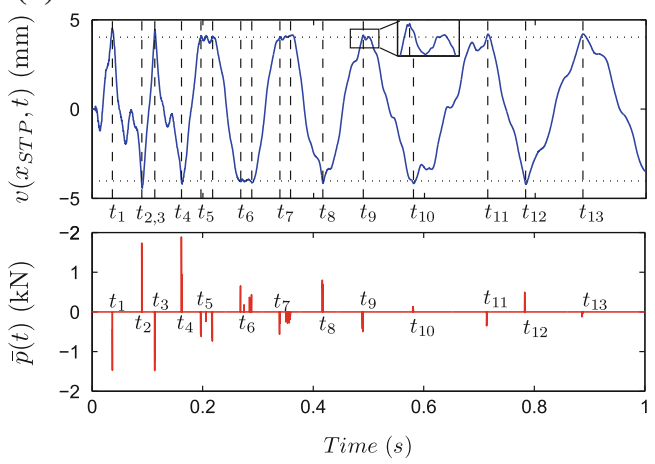

(b)
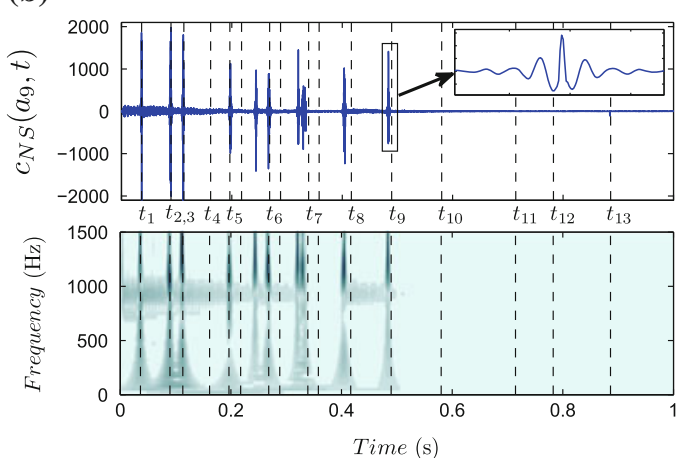

Fig. 7 Depiction of the vibro-impacts: a the displacement response of the VI beam simulated at position $x_{\text {STP }}$ and its corresponding impact loads on the beam from the rigid stops; $\mathbf{b}$ the nonsmooth component of the acceleration in Fig. 6 is decomposed via EMD analysis (Note that the dashed lines at $t=t_{k}, k=1, \ldots, 13$ imply the impact instants identified from the impact force $\bar{p}(t))$

effects by means of EMD analysis [38]. Typically, the nonsmooth part is computed as the first IMF with the help of masking and mirror-image signals [27]. The characteristics of the nonsmooth IMF were explored in previous works by relating them to Fourier series expansions of saw-tooth wave signals [41] and also by a partial-differential-equation-based sifting process [40] noting that EMD acts, in essence, as a dyadic filter bank.

Figure 7b depicts such a nonsmooth IMF for the acceleration signal in Fig. 6. Superposition of the impact instants identified from Fig. 7a illustrates that the isolated nonsmoothness agrees reasonably well with the time instants of vibro-impacts. We note that the numerical displacement was calculated from the reduced-order model through the assumed-modes method, which means that some other modes higher than 10th may need to be included to get a better match between the numerical simulations and experimental measurements. Some quantitative discrepancies prevail after $0.2 \mathrm{~s}$ with the current reduced-order model.

Now EMD is applied to the remaining smooth part of the acceleration signal after subtracting the nonsmooth IMF in Fig. 7b from the original acceleration in Fig. 6. The ten dominant IMFs are depicted in Fig. 8. By superimposing the vertical dashed line at each impact instant identified from Fig. 7a, one can at least qualitatively observe the effects of vibro-impacts on each IMF at position $x_{9}$; for example, the vibro-impacts seem to directly influence higher IMFs (above the 5th). Indeed, considering these higher frequency IMFs, we note linear dynamical behavior between consecutive vibro-impacts, in the form of exponentially decaying damped responses. On the other hand, lower IMFs do not seem to exhibit such straightforward patterns, implying that these IMFs may undergo more strongly nonlinear modal interactions and may be more significantly 

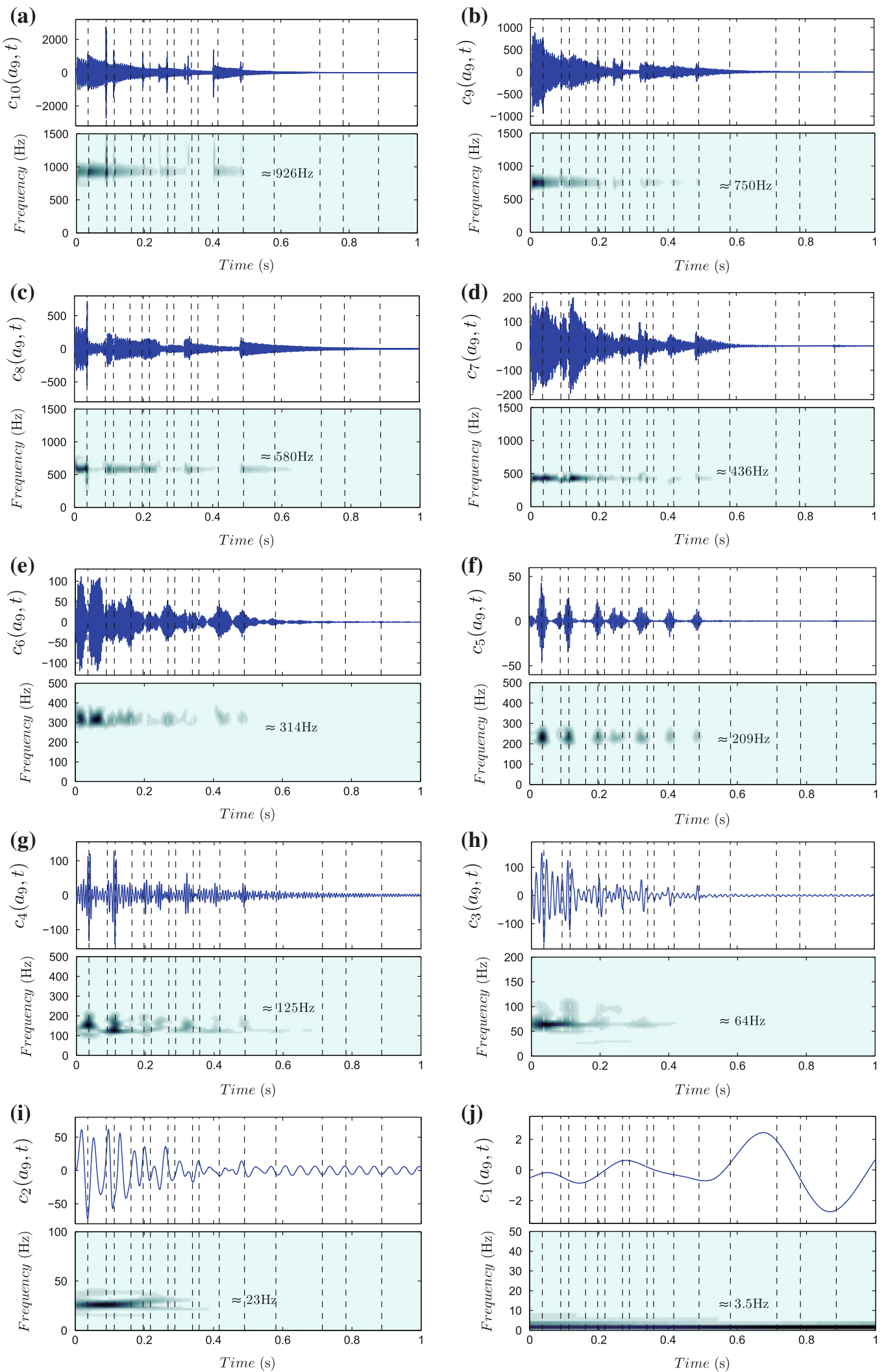

Fig. 8 The 10 dominant IMFs extracted from the acceleration response in Fig. 6: a through $\mathbf{j}$ sequentially depict the 10th to 1st IMFs, respectively (Note that the dashed lines imply the impact instants identified in Fig. 7) 
(a)

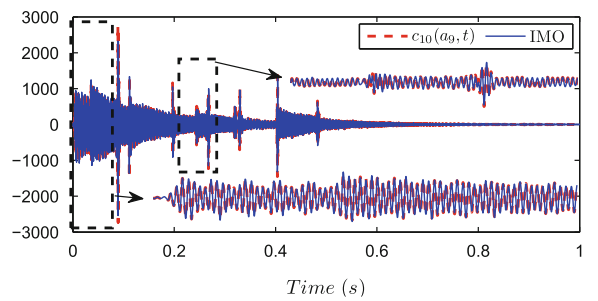

(b)

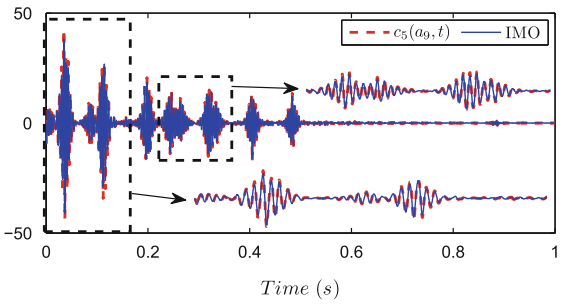

Fig. 9 Comparison of the IMFs in Fig. 8 with their corresponding IMO solutions: a 10th IMF; b 5th IMF

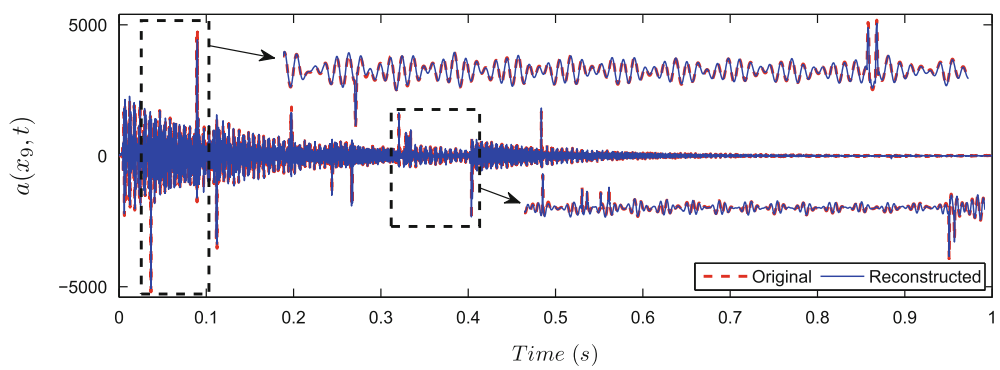

Fig. 10 Comparison of the reconstructed acceleration from the 10 IMO solutions plus the nonsmooth IMF with the original response in Fig. 6

influenced by the strong nonlinearities due the vibro-impacts. These aspects will be explored further in terms of the nonlinear modal interaction terms of the corresponding IMOs depicted in Figs. 11 and 13.

As in the linear beam, we can also establish a nonlinear interaction model (NIM) for the IMFs obtained in Fig. 8 in terms of a set of IMOs. Computing the nonlinear modal interaction forcing $\Lambda_{m}(t)$ [cf. Eq. (13)] from each IMF by means of the slow-flow correspondence (8), we solve the ten IMOs (11), respectively. Figure 9 compares the IMFs with the corresponding IMO solutions for 10th and 5th IMFs, which show good agreement. We sum all IMO solutions to reconstruct the original signal [cf. Eq. (20)], and this exhibits a perfect match as depicted in Fig. 10. That is, the NIM we established has been validated, so that it can be used to study the nonlinear dynamics of the VI beam (at position $x_{9}$ ) as an alternative reduced-order model.

As was discussed in Sect. 3.2, an understanding of the physical meaning of the slowly-varying complex forcing amplitude $\Lambda_{m}(t)$ in the ROM (or NIM for the VI beam) is essential, because the response obtained from the IMO in the ROM is strongly dictated by this nonhomogeneous forcing term, whereas the influence from the homogeneous part of the ROM is minimal. In other words, the ROM will predict linear (nonlinear) responses if the slowly-varying forcing term $\Lambda_{m}(t)$ exhibits linear (nonlinear) behaviors.

Now, the physical meaning of $\Lambda_{m}\left(a_{k}, t\right), m, k=1, \ldots, 10$, in the nonlinear dynamics of the VI beam can be explored by comparing it with that for the linear beam. We first note that the magnitude of $\Lambda_{m}\left(a_{9}, t\right)$ for all IMOs of the linear beam appears as almost a straight line on a logarithmic scale (cf. Fig. 11), which makes sense due to the form of Eq. (23). Similarly, $\left|\Lambda_{m}\left(a_{9}, t\right)\right|$ for the VI beam can also exhibit linearity with the same slope on average on a logarithmic scale as in the case of the linear beam, but such a linear pattern appears only in between impact instants and, in particular, when $m \geq 6$ (cf. Fig. 11a for the 10th IMO). The trajectory of $\Lambda_{10}\left(a_{9}, t\right)$ in the complex plane for the linear beam appears as a single, monotonic, decaying pattern (i.e., time-like behavior on a logarithmic scale), which implies no modal coupling or interactions in the ROM by means of Eq. (11), as was discussed in Sect. 3.2. The trajectory of $\Lambda_{10}\left(a_{9}, t\right)$ in the complex plane for the VI beam also exhibits such monotonic behavior but only in between vibro-impacts (denoted by the intervals $\left.I_{n}, n=1,2, \ldots\right)$; the role of the vibro-impacts is to cause phase shifts of the slowly-varying forcing $\Lambda_{10}\left(a_{9}, t\right)$ at the instants of vibro-impacts. On the other hand, the slowly-varying complex forcing function for the 4th IMO of the VI beam does not exhibit any linear behavior but only a slowly-varying wavy envelope regardless of vibro-impacts (cf. Fig. 11b). Such wavy patterns in the plot of $\left|\Lambda_{4}\left(a_{9}, t\right)\right|$ indicate that certain modal interactions occur through nonlinear resonant conditions such as internal resonances or resonance captures [31]. Also, nonlinear modal interactions are evidenced by the spiral (or non-time-like) patterns of the trajectory in the complex plane. 

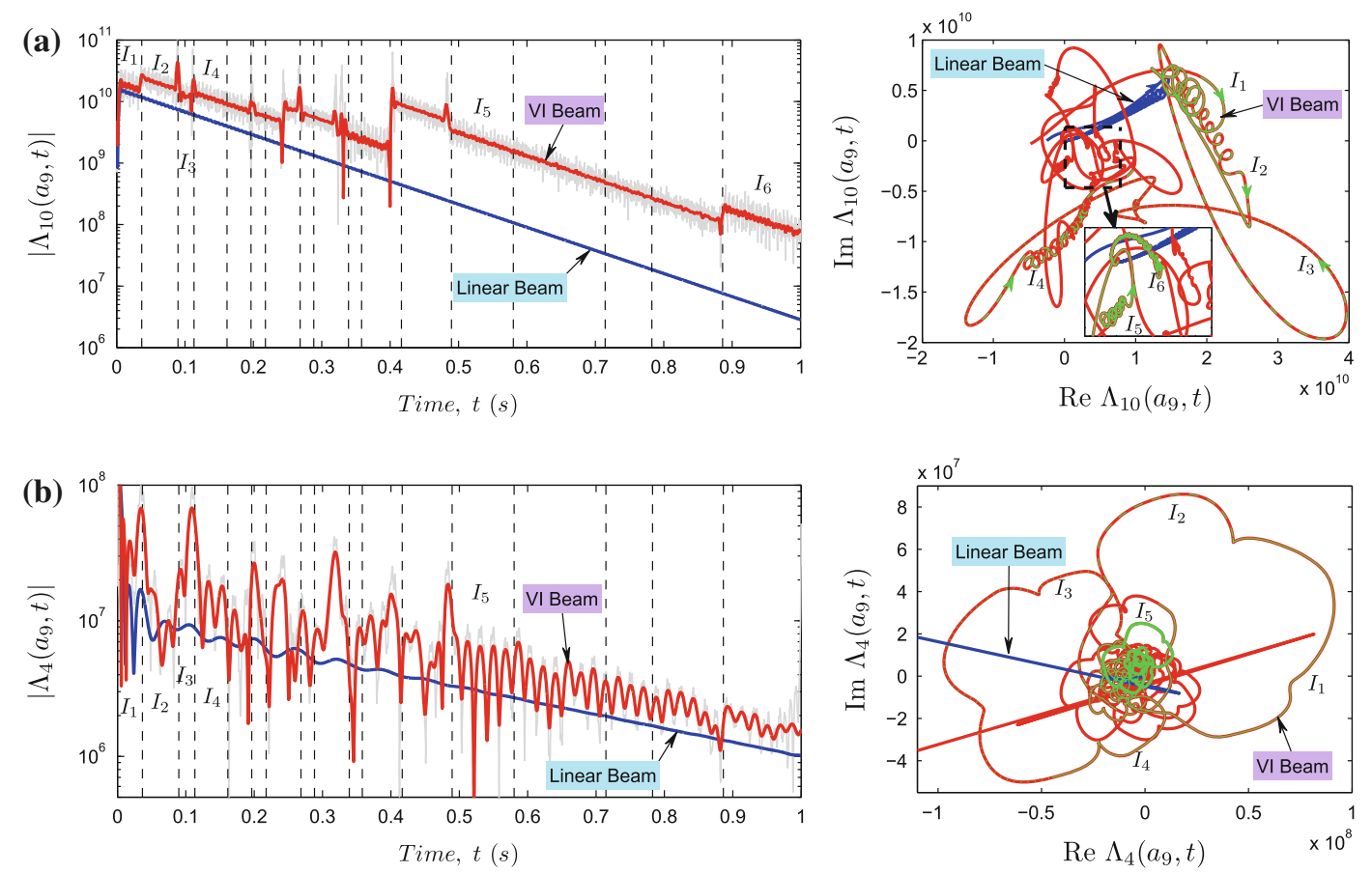

Fig. 11 Comparison of the slowly-varying forcing functions $\Lambda_{m}\left(a_{9}, t\right)$ : a $m=10$ (i.e., 10th IMO) and $\mathbf{b} m=4$ (i.e., 4 th IMO). Note that the dashed lines imply the impact instants identified in Fig. 7

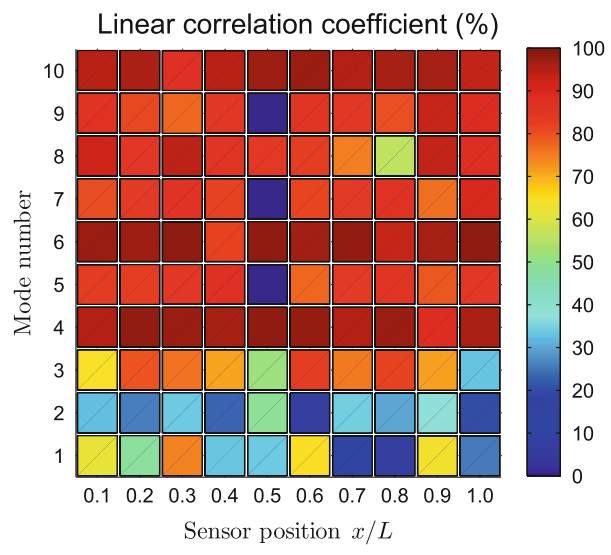

Fig. 12 Linear correlation coefficient (\%) between the slowly-varying forcing functions $\Lambda_{m}\left(a_{k}, t\right), m, k=1, \ldots, 10$, where $m$ and $k$ denote the mode number and accelerometer position, respectively, of the linear and VI beams

From these two typical examples, we may conjecture the following: Whereas the higher IMOs (i.e., the IMOs associated with higher frequency components) tend to maintain their linear dynamics in between impacts (although the overall dynamics is strongly nonlinear), the lower IMOs exhibit strongly nonlinear modal interactions independent of vibro-impact patterns. The role of vibro-impacts is just to exert broadband impulsive excitations on the linear beam causing instantaneous phase shifts in the higher IMOs.

To verify this conjecture, we first compute Pearson's linear correlation coefficient [42] for the slowly-varying complex forcing amplitudes $\Lambda_{m}\left(a_{k}, t\right), m, k=1, \ldots, 10$, of all IMOs for the linear and VI beams at all the positions along the beam. This correlation coefficient is widely utilized in statistics as a measure of the linear dependence between two variables, and a Matlab command, 'corr.m', was used in this work. Figure 12 depicts the interpolated contour map of the absolute value of the linear correlation coefficient for each mode number along the beam span. Note that by 'mode number' $m$ in Fig. 12, we mean the IMO which is associated with the $m$ th linear mode. From these simple calculations, we find that the IMOs higher than the 4th possess strong linear dependence (linear correlation coefficient above 90\%) between the linear and VI responses of 

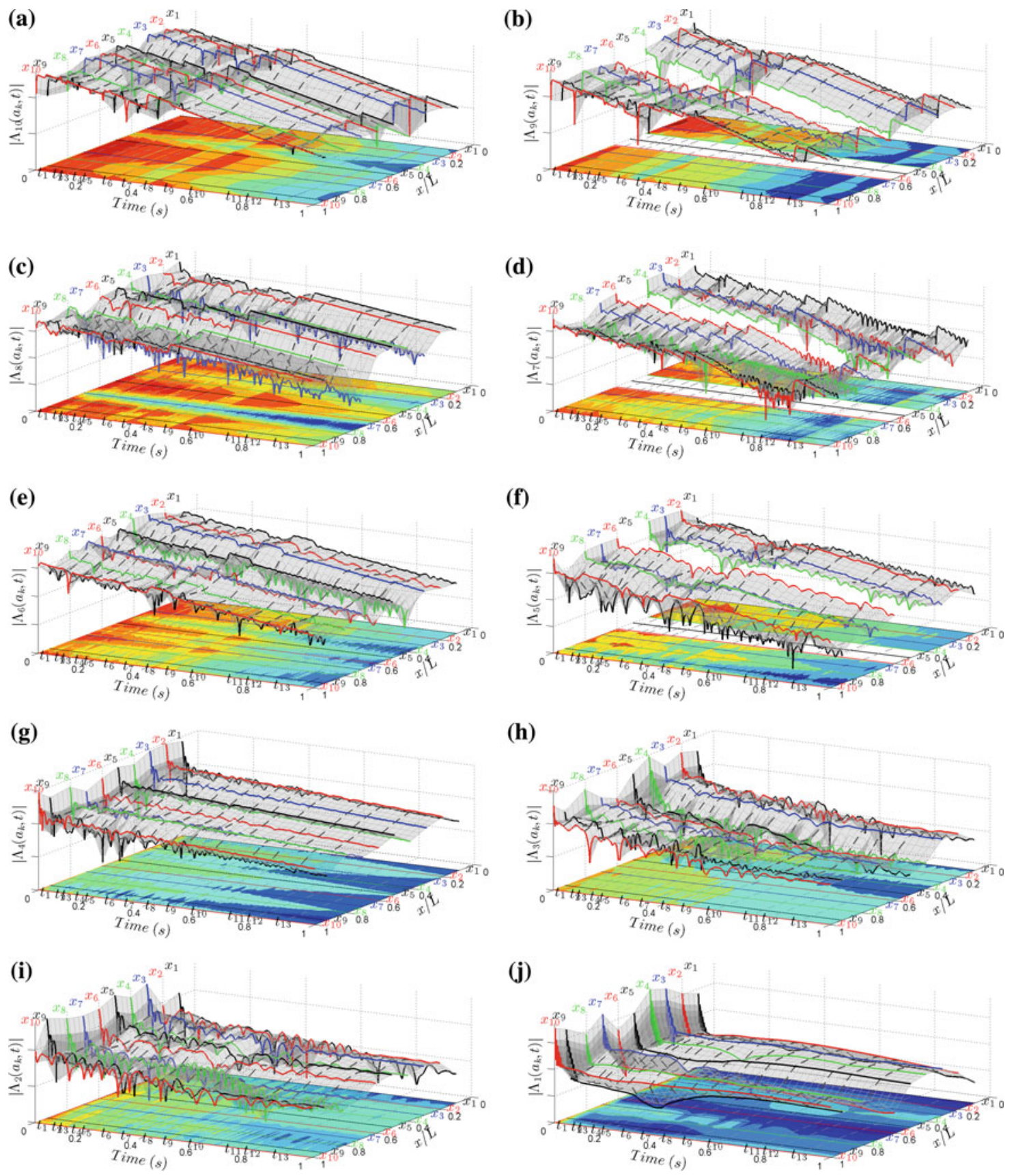

Fig. 13 The spanwise magnitudes of nonlinear modal interaction for the VI beam: a through $\mathbf{j}$ sequentially depict the 10th to 1 st IMFs, respectively (Note that the logarithm with base 10 is taken for $\left|\Lambda_{m}\left(a_{k}, t\right)\right|$, and that the sections at $t=t_{n}, n=1, \ldots, 9$, denote some of the impact instants identified in Fig. 7)

the beam, regardless of the position along the beam. Again, it is noted that the low correlation for the 3rd, 5th, 7th and 9th IMOs at the midspan of the beam is due to the fact that the position is very close to one of the nodes for the respective linear modes. Similar explanations can be given for the 5th and 7th IMOs at position $x_{9}$, and for the 8th IMO at position $x_{8}$. Therefore, the aforementioned conjecture is confirmed by means of the linear correlation coefficients between $\left|\Lambda_{m}\left(a_{k}, t\right)\right|$ (and hence the corresponding IMO responses) for the linear and VI beams. That is, vibro-impacts do not significantly alter the linear dynamics for the higher modes (typically, higher than 4th), but they significantly affect the lower modes through strongly nonlinear modal interactions. This result agrees with Cusumano's previous work [22], where the topological characterization of the spatial 
structure of the VI beam vibrations was studied by means of the two-point spatial correlation (i.e., correlation dimension) and POD. In particular, the estimate for the correlation dimension of the VI dynamics obtained was lower than but near 4, which dictates that a low-dimensional model can capture the overall complicated, chaotic-like VI beam dynamics. Furthermore, if such complicated dynamics can be captured by a low-dimensional model with several lower IMOs, then energy transfers (or cascades) from the higher to the lower modes through certain nonlinear modal interactions, such as internal resonances, may be responsible [22].

While the linear correlation coefficient provides excellent physical insights into the VI beam dynamics, we note that it can be regarded as a 'static' global measure; that is, it does not contain any information regarding the temporal variations of the vibro-impacts throughout the beam. A high linear correlation coefficient for certain IMOs at some position may indicate a strong linear dependence between the VI beam and the underlying linear beam, and imply that the corresponding IMO of the VI beam behaves linearly for that position. Nonetheless, this will not be apparent in the local dynamics (e.g., propagation and/or localization of the effects of nonlinear modal interactions caused by vibro-impacts, such as the temporal localization of the nonlinear dynamics for the 7th IMO). In particular, the linear correlation becomes a poor measure when the issue of observability is involved (e.g., all the odd-number IMOs higher than 3rd at position $x_{5}$ in Fig. 12).

Hence, we construct a different set of maps for the spatio-temporal variations of $\left|\Lambda_{m}\left(a_{k}, t\right)\right|, m, k=$ $1, \ldots, 10$, depicted in Fig. 13 for all computed IMOs. We note that, since the position $x_{5}$ is very close to one of the nodes for the 5th, 7th and 9th linear modes, no meaningful IMFs could be obtained for this measuring position, creating discontinuous surface plots in Fig. 13b, d and f, respectively. Linear logarithmic decrement of $\left|\Lambda_{m}\left(a_{k}, t\right)\right|$ in time between vibro-impacts indicates that linear dynamics between vibro-impacts prevails for IMOs higher than the 3rd (cf. Fig. 13a-g) for the VI beam dynamics. On the other hand, we can confirm that modes lower than the 4th are significantly influenced by vibro-impacts (cf. Fig. 13h-j). That is, strong, wavy, wrinkled spatio-temporal distributions of $\left|\Lambda_{m}\left(a_{k}, t\right)\right|$ can be observed from the 3-dimensional surface plot, as compared to the almost flat distributions of $\left|\Lambda_{m}\left(a_{k}, t\right)\right|$ with simultaneous occurrences of spikes due to vibro-impacts for the higher IMOs. The lower IMOs also appear as high-frequency, smudged images of recurrent nested contours in the 2-dimensional projection, and accordingly, the impact patterns associated with vibro-impacts are hard to read and no longer simultaneous at all sensing positions.

Finally, we remark that not all higher IMOs behave completely linearly in between vibro-impacts. Whereas the spatio-temporal variations of $\left|\Lambda_{m}\left(a_{k}, t\right)\right|$ for the 9th and 10th IMOs are almost globally linear (evidenced by the formation of nearly rectangular strips in the contour plot on the $(x, t)$ plane, neglecting changes in their absolute magnitudes), those for the 4th-8th IMOs manifest spatially-localized nonlinear behavior, in particular, at position $x_{9}$ where vibro-impacts from the rigid stops strongly affect the beam dynamics. The slowly-varying complex forcing at positions $x_{3}$ and $x_{8}$ for the 8th IMO, and at position $x_{4}$ for the 5th IMO also demonstrate spatially-localized nonlinear effects on the VI beam. Furthermore, the 7th IMO even exhibits temporal localization of the nonlinear dynamics in between the impact instants $t_{11}$ and $t_{13}$ in Fig. 13d.

\section{Comparison with results of experimental data analysis}

In this section, we compare the NSI analysis of the numerical data in Sect. 3 with experimental measurements for the VI beam. Note that comparisons in this section will remain qualitative, because the impulsive excitations by an impact hammer for the experiments were different than the impulsive excitations used in the numerical simulation. That is, a plastic tip was put on the impact hammer in the experiments, whereas an aluminum tip was considered in the numerical simulations. Use of such a plastic tip on the impact hammer limits the frequency bandwidth of the excitation, whereas an aluminum tip broadens the excitation bandwidth. A more complete NSI analysis of the experimental data will be discussed in a companion paper [43], so here we provide only a preliminary qualitative comparison between computational and experimental results.

The procedures for NSI of the experimental VI beam responses are the same as those for the numerical data. We perform EMD analysis of the ten acceleration measurements respectively at the ten sensing positions $\left(x_{1}\right.$ through $\left.x_{10}\right)$ along the beam span. For the purpose of comparison, NSI of the acceleration at position $x_{9}$ is our primary interest in the current work. The 10th and 5th IMFs of this experimental acceleration response are depicted in Fig. 14a and b, respectively. Comparing them with those depicted in Fig. 8, we find qualitative similarity. That is, the 10th IMF exhibits multiple exponential decays initiated at every vibro-impact, whereas the 5th IMF seems to exhibit strong nonlinear modal interactions. Indeed, this observation is qualitatively consistent with that for the numerical data.

Then, we establish a nonlinear interaction model (NIM) as a set of IMOs [cf. Eq. (11)], whose solutions are verified and validated to match the respective IMFs. Furthermore, we confirm completeness of the IMO 
(a)
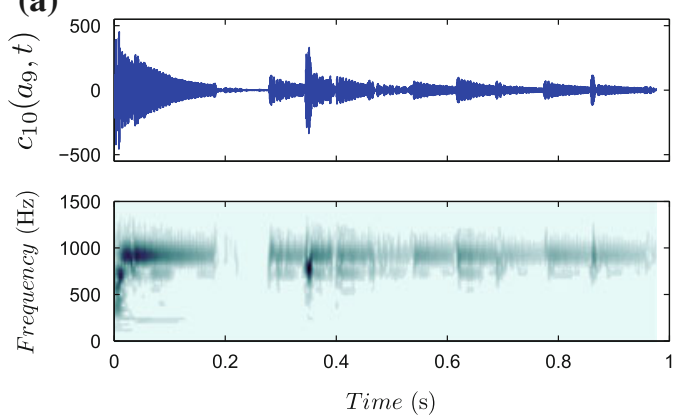

(b)
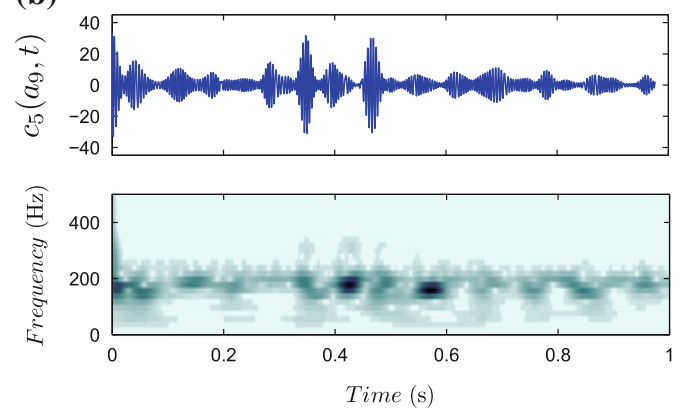

Fig. 14 IMFs extracted from the experimentally measured acceleration at the sensing position $x_{9}$ : a 10th IMF (cf. Fig. 8a); b 5th IMF (cf. Fig. 8f)

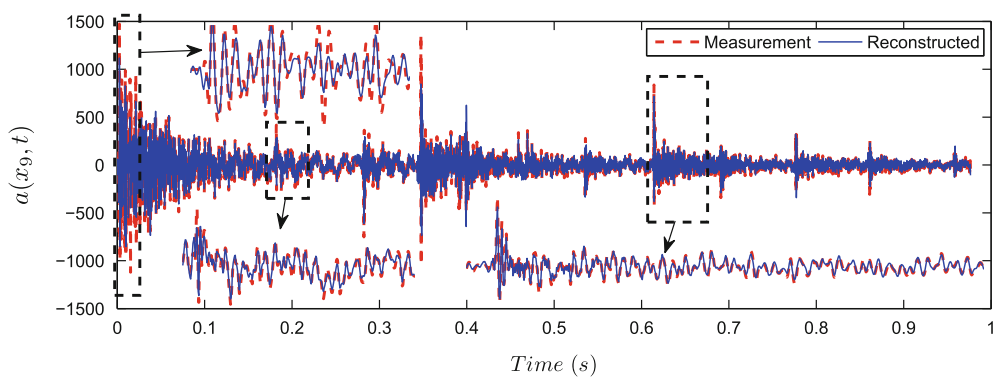

Fig. 15 Comparison of the reconstructed acceleration from the ten (smooth) IMO solutions with the original experimental response measured at position $x_{9}$

(a)

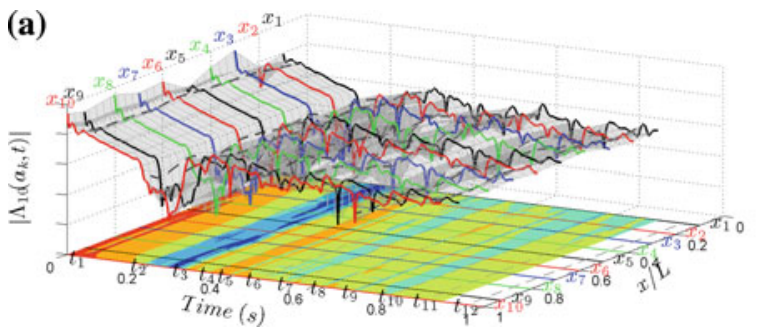

(b)

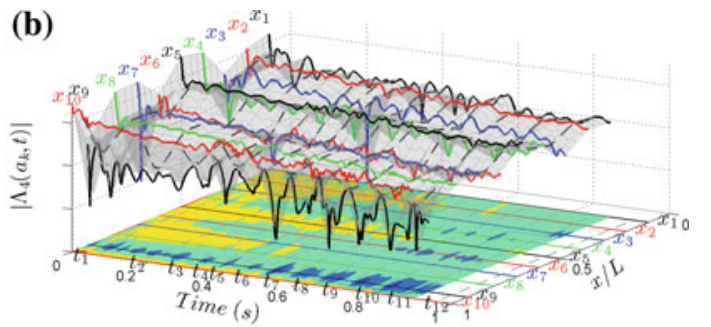

Fig. 16 The spatio-temporal variations of $\Lambda_{m}\left(a_{9}, t\right)$ the VI beam from experimental measurements: a $m=10$ (i.e., 10th IMF); b $m=5$ (i.e., 5th IMF)

solutions as a basis with which the given measurement can be expanded. This is demonstrated in Fig. 15. Note that the apparent discrepancies between the measured and reconstructed acceleration signals are due to the fact that the nonsmooth IMF was not included when reconstructing the original signal.

Finally, Fig. 16a and $\mathrm{b}$ depict the spatio-temporal variations of $\left|\Lambda_{m}\left(a_{k}, t\right)\right|, k=1, \ldots, 10$, respectively, for the 10th $(m=10)$ and 5th $(m=5)$ IMFs from experimental measurements at the 10 positions along the beam. As was discussed in Sect. 3.3, from the NSI of the numerical data, the 10th IMO manifests linear dynamics in between vibro-impacts (cf. Fig. 13a), whereas the 5th IMO does not (cf. Fig. 13g). For the 10th (or higher) IMO, formation of the flat strips is more evident, compared to that of the numerical data; moreover, the influences of vibro-impacts occur simultaneously along the whole beam. On the other hand, the 5th (or lower) IMO exhibits linear dynamics between instants of vibro-impacts, except for the position $x_{9}$ where strong effects due to vibro-impacts from the rigid stops exist in the beam dynamics. Also, the measurements at positions such as $x_{1}$ and $x_{3}$ exhibit localized nonlinear modal interactions evidenced by the wavy, wrinkled surface plot. Overall, NSI of the experimental results demonstrates dynamical behaviors qualitatively similar to those observed in the numerical results. The NSI of the experimental results will be further discussed in a companion paper [43]. 


\section{Concluding remarks}

We studied the dynamics of a cantilever beam with two symmetric rigid stops with prescribed clearances by performing nonlinear system identification (NSI) based on the correspondence between analytical and empirical slow-flow dynamics. Performing empirical mode decomposition (EMD) analysis of the numerically computed acceleration responses at ten, almost evenly-spaced, spanwise positions along the beam, we constructed sets of intrinsic modal oscillators at different time scales of the dynamics. In particular, the EMD analysis can separate nonsmooth effects due to vibro-impacts between the beam and the rigid stops from the underlying smooth dynamics of the flexible beam, so that nonlinear modal interactions can be explored only based on the remaining smooth components. Then, we established nonlinear interaction models (NIMs) for the respective intrinsic mode oscillations, where the NIMs invoke slowly-varying forcing amplitudes (or nonlinear modal interaction terms) that can be computed from empirical slow-flows and directly dictate nonlinear modal interactions between different-scale dynamics. By comparing the spatio-temporal variations of the nonlinear modal interactions for the vibro-impact beam and the corresponding linear beam model, we demonstrated that vibro-impacts significantly influence the lower intrinsic mode functions through strongly nonlinear modal interactions, whereas the higher modes tend to retain their linear dynamics between impacts. Also, computation of linear correlation coefficients as measures for linear dependence between the dynamics of the linear and VI beams manifested the same results but only with spatial information about this correlation. A preliminary comparison of numerical and experimental NSI results demonstrated qualitative similarity between the experimental and numerical simulations. Further analysis of the experimental results will be discussed in a companion paper [43].

Acknowledgments This work was supported in part by the National Science Foundation of United States through Grants Number CMMI-0927995 and CMMI-0928062.

\section{References}

1. Ewins, D.J.: Modal Testing: Theory and Practice. Research Studies Press, UK (1990)

2. Brandon, J.A.: Some insights into the dynamics of defective structures. Proc. Inst. Mech. Eng. Part C: J. Mech. Eng. Sci. 212, 441-454 (1998)

3. Kerschen, G., Golinval, J.-C., Vakakis, A.F., Bergman, L.A.: The method of proper orthogonal decomposition for order reduction of mechanical systems: an overview. Nonlinear Dyn. 41, 147-170 (2005)

4. Kerschen, G., Worden, K., Vakakis, A.F., Golinval, J.-C.: Past, present and future of nonlinear system identification in structural dynamics. Mech. Syst. Signal Process. 20, 505-592 (2005)

5. Feeny, B.F., Kappagantu, R.: On the physcal interpretation of proper orthogonal modes in vibrations. J. Sound Vib. 211, 607-616 (1998)

6. Kerschen, G., Golinval, J.C.: Physical interpretation of the proper orthogonal modes using the singular value decomposition. J. Sound Vib. 249, 849-865 (2002)

7. Bellizzi, S., Sampaio, R.: POMs analysis of randomly vibrating systems obtained from Karhunen-Loève expansion. J. Sound Vib. 297, 774-793 (2006)

8. Allison, T.C., Miller, A.K., Inman, D.J.: A deconvolution-based approache to structural dynamics system identification and response prediction. J. Vib. Acoust. 130, 031010 (2008)

9. Chelidze, D., Zhou, W.: Smooth orthogonal decomposition-based vibration mode identification. J. Sound Vib. 292, 461-473 (2006)

10. Silva, W.: Identification of nonlinear aeroelastic systems based on the Volterra theory: progress and opportunities. Nonlinear Dyn. 39, 25-62 (2005)

11. Li, L.M., Billings, S.A.: Analysis of nonlinear oscillators using Volterra series in the frequency domain. J. Sound Vib. 330, 337-355 (2011)

12. Mariani, S., Ghisi, A.: Unscented Kalman filtering for nonlinear structural dynamics. Nonlinear Dyn. 49, 131-150 (2007)

13. Masri, S., Caughey, T.: A nonparametric identification techanique for nonlinear dynamic systems. J. Appl. Mech. 46, 433-441 (1979)

14. Leontaritis, I.J., Billings, S.A.: Input-output parametric models for nonlinear systems. Part I. Deterministic nonlinear systems; Part II. Stochastic nonlinear systems. Int. J. Control 41, 303-328; 329-344 (1985)

15. Thothadri, M., Casas, R.A., Moon, F.C., D’Andrea, R., Johnson, C.R. Jr.: Nonlinear system identification of multi-degreeof-freedom systems. Nonlinear Dyn. 32, 307-322 (2003)

16. Feldman, M.: Non-linear system vibration analysis using Hilbert transform-I. Free vibration analysis method 'FREEVIB'; II. Forced vibration analysis method 'FORCEVIB'. Mech. Syst. Signal Proces. 8, 119-127; 309-318 (1994)

17. Feldman, M.: Time-varying vibration decomposition and analysis based on the Hilbert transform. J. Sound Vib. 295, 518-530 (2006)

18. Ma, X., Azeez, M.F.A., Vakakis, A.F.: Non-linear normal modes and non-parametric system identification of non-linear oscillators. Mech. Syst. Signal Process. 14, 37-48 (2000) 
19. Georgiou, I.: Advanced proper orthogonal decomposition tools: using reduced order models to identify normal modes of vibration and slow invariant manifolds in the dynamics of planar nonlinear rods. Nonlinear Dyn. 41, 69-110 (2005)

20. Galvanetto, U., Surace, C., Tassotti, A.: Structural damage detection based on proper orthogonal decomposition: experimental verification. AIAA J. 46, 1624-1630 (2008)

21. Cusumano, J.P., Bae, B.-Y.: Period-infinity periodic motions, chaos, and spatial coherence in a 10 degree of freedom impact oscillator. Chaos, Solitons Fractals 3, 515-535 (1993)

22. Cusumano, J.P., Sharkady, M.T., Kimble, B.W.: Experimental measurements of dimensionality and spatial coherence in the dynamics of a flexible-bea impact oscillator. Philos. Trans. R. Soc. Ser. A 347, 421-438 (1994)

23. Ritto, T.G., Buezas, F.S., Sampaio, R.: A new measure of efficiency for model reduction: application to a vibroimpact system. J. Sound Vib. 330, 1977-1984 (2011)

24. Azeez, M.F.A., Vakakis, A.F.: Proper orthogonal decomposition (POD) of a class of vibroimpact oscillations. J. Sound Vib. 240, 859-889 (2001)

25. Lee, Y.S., Vakakis, A.F., McFarland, D.M., Bergman, L.A.: A global-local approach to system identification: a review. Struct. Control Health Monit. 17, 742-760 (2010)

26. Huang, N., Shen, Z., Long, S., Wu, M., Shih, H., Zheng, Q., Yen, N.-C, Tung, C., Liu, H.: The empirical mode decompostion and the Hilbert spectrum for nonlinear and non-stationary time series analysis. Proc. R. Soc. Lond. Ser. A. Math. Phys. Sci. 454, 903-995 (1998)

27. Lee, Y.S., Tsakirtzis, S., Vakakis, A.F., Bergman, L.A., McFarland, D.M.: Physics-based foundation for empirical mode decomposition. AIAA J. 47, 2938-2963 (2009)

28. Lee, Y.S., Tsakirtzis, S., Vakakis, A.F., McFarland, D.M., Bergman, L.A.: A time-domain nonlinear system identification method based on multiscale dynamic partitions. Meccanica 46, 625-649 (2010)

29. Lee, Y.S., Vakakis, A.F., McFarland, D.M., Bergman, L.A.: Nonlinear system identification of the dynamics of aeroelastic instability suppression based on targeted energy transfers. Aeronaut. J. 114, 61-82 (2010)

30. Tsakirtzis, S., Lee, Y.S., Vakakis, A.F., Bergman, L.A., McFarland, D.M.: Modeling of nonlinear modal interactions in the transient dynamics of an elastic rod with an essentially nonlinear attachment. Commun. Nonlinear Sci. Numer. Simul. 15, 2617-2633 (2010)

31. Dawes, J.H.P.: Review: the emergence of a coherent structure for coherent structures: localized states in nonlinear systems. Philos. Trans. R. Soc. Ser. A 368, 3519-3534 (2010)

32. Chati, M., Rand, R., Mukherjee, S.: Modal analysis of a cracked beam. J. Sound Vib. 207, 249-270 (1997)

33. Chen, H.G., Yan, Y.J., Jiang, J.S.: Vibration-based damage detection in composite wingbox structures by HHT. Mech. Syst. Signal Process. 21, 307-321 (2007)

34. Mane, M.: Experiments in Vibro-Impact Beam Dynamics and a System Exhibiting a Landau-Zener Quantum Effect. MS Thesis (unpublished), Univeristy of Illinois at Urbana-Champaign (2010)

35. Blevins, R.D.: Formulas for Natural Frequency and Mode Shape. Krieger, New York (1995)

36. Manevitch, L.: The description of localized normal modes in a chain of nonlinear coupled oscillators using complex variables. Nonlinear Dyn. 25, 95-109 (2001)

37. Lochak, P., Meunier, C.: Multiphase Averaging for Classical Systems: With Applications to Adiabatic Theorems. Springer, New York (1988)

38. Lee, Y.S., Nucera, F., Vakakis, A.F., McFarland, D.M., Bergman, L.A.: Periodic orbits and damped transitions of vibro-impact dynamics. Phys. D 238, 1868-1896 (2009)

39. Nordmark, A.B.: Existence of periodic orbits in grazing bifurcations of impacting mechanical oscillators. Nonlinearity 14, 1517-1542 (2001)

40. Deléchelle, E., Lemoine, J., Niang, O.: Empirical mode decomposition: an analytical approach for sifting process. IEEE Signal Process. Lett. 12, 764-767 (2005)

41. Lee, Y.S., Chen, H., Vakakis, A.F., McFarland, D.M., Bergman, L.A.: Nonlinear system identification of vibro-impact nonsmooth dynamical systems (AIAA-2011-2067). In: 52nd AIAA Structures, Structural Dynamics and Materials Conference, Denver, Colorado, 4-7 April 2011 (2011)

42. Gibbons, J.D.: Nonparametric Statistical Inference. 2nd edn. M. Dekker, New York (1985)

43. Chen, H., Kurt, M., Lee, Y.S., McFarland, D.M., Bergman, L.A., and Vakakis, A.F.: System identification of a vibro-impact beam with a view toward structural health monitoring. Exp. Mech. (submitted) 